\title{
Searching for diquarks in hadrons
}

\section{Constantia Alexandrou}

Department of Physics, University of Cyprus, CY-1678 Nicosia, Cyprus

E-mail: alexand@ucy.ac.cy

\section{Philippe de Forcrand}

Institute for Theoretical Physics, ETH Zürich, CH-8093 Zürich, Switzerland CERN, Physics Department, TH Unit. CH-1211 Genève 23, Switzerland

E-mail: forcrandephys.ethz.ch

\section{Biagio Lucini*}

Institute for Theoretical Physics, ETH Zürich, CH-8093 Zürich, Switzerland

E-mail: lucini@phys.ethz.ch

Since the early days of QCD, it has been argued that inside hadrons quarks organise into substructures. Among those, popular pictures of various phenomena (including exotica and colour superconductivity) give a prominent role to diquarks, i.e. to colour antitriplet combinations of two quarks. Using a gauge-invariant setup, which combines the diquark with a static quark, we study spatial correlations of the two light quarks inside the diquark. After illustrating the setup, we discuss our first results for both the scalar ("good") and the spin one ("bad") diquark channels. In a regime in which $m_{\pi} \simeq 830 \mathrm{MeV}$, our data show unambiguously that a scalar diquark forms with a size of $\simeq 0.9 \mathrm{fm}$. For the vector diquark we are able to put a lower bound of 4.1(7) fm on its size. We also investigate the mass splitting between the good and the bad diquark; our findings are compatible with phenomenologically inspired predictions for this quantity.

XXIIIrd International Symposium on Lattice Field Theory

25-30 July 2005

Trinity College, Dublin, Ireland

\footnotetext{
* Speaker.
} 


\section{Introduction}

The static quark model has provided a rather accurate description of hadronic states over the last four decades. This is a rather simple model, since it assumes that quarks inside hadrons are uncorrelated. Correlations among quarks could however explain remaining features of the spectrum that look incompatible with the simple-minded static quark model. In fact a natural possibility is that two quarks combine into a colour antitriplet representation. This correlation is energetically favourable, since at large distances the resulting chromoelectric field is simply that of an antiquark. Colour antitriplet combinations of two quarks are called diquarks.

Diquarks have been discussed since the early days of QCD (see Ref. [1] for a review). Interest in this topic has been recently revived by the increased attention devoted to the high density phase of QCD [2] and to the possible existence of exotic particles [3, 4], since in both cases diquarks could play an important role. Diquark-inspired phenomenological models usually start from the assumption that those bound states exist. However this assumption needs to be justified from first principles. As usual the lattice is an excellent framework for such $a b$ initio calculations. By using a gauge-invariant formalism that enables us to study their structure, we show unambiguously that diquark states do form and present preliminary results for the mass splittings between the scalar and vector diquark channels. Our calculation is done in the quenched theory using Wilson fermions and quark masses that correspond to pion mass in the range $830-1000 \mathrm{MeV}$.

\section{Probing the structure of a diquark}

Diquark operators have been discussed in [5]. For convenience, the quantum numbers and the colour and flavour representations of the simplest diquark operators are reported in Table. 1 . In this work we focus on the $\bar{q}_{C} \gamma_{0} \gamma_{5} q$ (the scalar diquark) and the $\bar{q}_{C} \vec{\gamma}_{q}$ (the vector diquark) combinations. Effective colour-spin hamiltonian arguments suggest that the spin zero channel is enhanced with respect to the spin one combinations [5]. For this reason the scalar and the vector diquarks are also referred to respectively as the "good" and the "bad" diquarks.

\begin{tabular}{|c|c|c|c|}
\hline$J^{P}$ & Colour & Flavour & Operator \\
\hline $0^{+}$ & $\overline{3}$ & $\overline{3}$ & $\bar{q}_{C} \gamma_{5} q, \bar{q}_{C} \gamma_{0} \gamma_{5} q$ \\
$1^{+}$ & $\overline{3}$ & 6 & $\bar{q}_{C} \vec{\gamma}_{q}, \bar{q}_{C} \sigma^{0 i} q$ \\
\hline
\end{tabular}

Table 1: The structure of the simplest diquark operators $\left(\bar{q}_{C}\right.$ is the conjugated spinor of $\left.q\right)$.
We now want to investigate whether a diquark bound state forms. The issue is complicated by the fact that diquarks are coloured objects. In order to formulate the problem in a gaugeinvariant way, we combine the diquark with a static quark in a colour singlet representation. Far from the source, the influence of the static chromoelectric field becomes less important and the physics of the diquark emerges. The static-light

baryon obtained by combining a static colour source with a light diquark can be studied with the density-density correlator method [6], which provides a gauge-invariant technique for studying quark distributions inside a hadron. This method has been recently used in Ref. [7, 8] for studying the structure of various hadronic states.

Our setup is sketched in Fig. 1. At Euclidean time $t=0$ we create a static-light baryon in which the two light quarks are combined into a candidate diquark channel (either scalar or vector) and we 


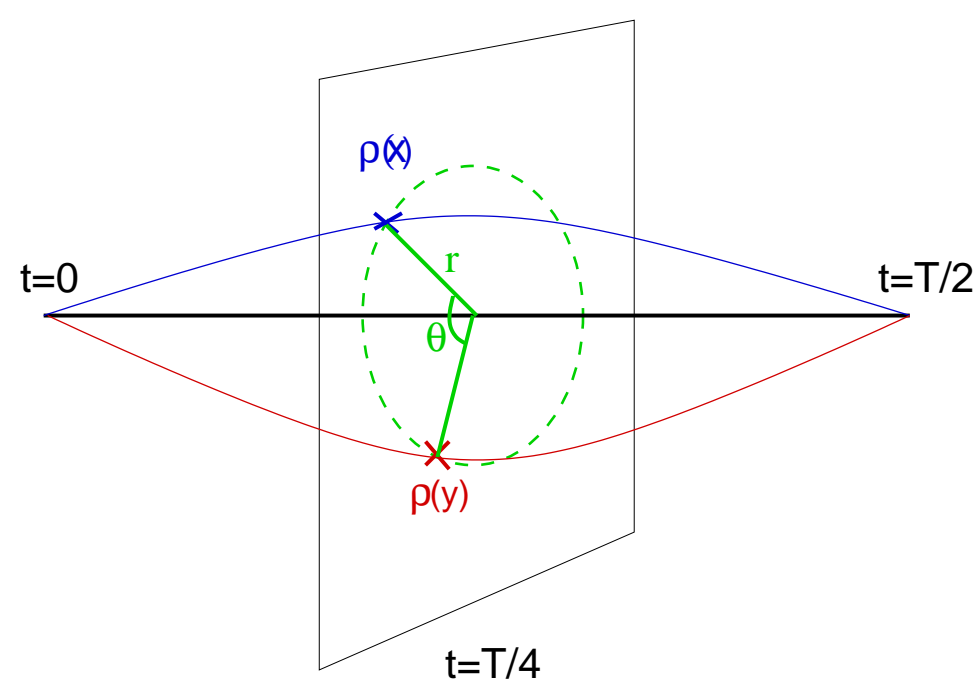

Figure 1: Setup for the computation of the density-density correlators.

let this state propagate to time $t=T / 2$ ( $T$ is the extension of the lattice in the temporal direction), where it is annihilated. Far from the source and the sink, at a fixed Euclidean time $t(t=T / 4$ in Fig. 1) where the hadron has relaxed to its ground state, we study the density-density correlator of the two light quarks

$$
C(\theta, r)=\int\left\langle H\left|\rho_{u}\left(\vec{r}_{u}\right) \rho_{d}\left(\vec{r}_{d}\right)\right| H\right\rangle \delta\left(\left|\vec{r}_{u}\right|-r\right) \delta\left(\left|\vec{r}_{d}\right|-r\right) \delta\left(\hat{r}_{u} \cdot \hat{r_{d}}-\cos \theta\right) \mathrm{d} \vec{r}_{d} \mathrm{~d} \vec{r}_{u},
$$

where the subscripts $u$ and $d$ refer respectively to the up and down quarks, $\rho_{q}=: \bar{q} \gamma_{0} q:$ and $\vec{r}_{u}$ and $\vec{r}_{d}$ are the lattice vector coordinates of the light quarks with respect to the static source. To make the analysis of our results clearer, we consider correlators for $\left|\vec{r}_{u}\right|=\left|\vec{r}_{d}\right|=r$ : the two light quarks are on the same spherical shell at distance $r$ from the static source. Since the system is spherically symmetric, $C$ only depends on $r$ and the angle $\theta=\arccos \left(\hat{r}_{u} \cdot \hat{r}_{d}\right)$. If a diquark state is favored by QCD dynamics, one would expect that $C(\theta, r)$ is enhanced at small values of $\theta$, while if the quarks are uncorrelated the distribution is flat in $\cos \theta$. On a lattice the angular distribution is distorted by discretisation artifacts, especially for small values of $r$. To suppress those effects we have normalized our distributions to that obtained from uncorrelated objects on the same lattice.

We have measured $C(\theta, r)$ on 200 quenched configurations at $\beta=6.0$ taken from the NERSC archive $^{1}$ using two degenerate flavours of Wilson fermions at $\kappa=0.153$. Those lattice parameters correspond to $m_{\pi} \simeq 830 \mathrm{MeV}$ and to a lattice spacing $a \simeq 0.097$ fermi. The results are obtained by binning in $\cos \theta$ within shells of "thickness" $\delta r=0.1 r$ (so that $\left|\vec{r}_{u}\right| /\left|\vec{r}_{d}\right|=1 \pm 0.1$ ). The magnitude of the correlator decays exponentially with the distance $r$ from the static quark, with a decay length of $0.90(2) a$ (corresponding to a mass of 2.25(5) GeV). Despite this, the signal never dies into noise and we are able to study the density-density correlator up to the maximal allowed distance $r=8 a$ on our $16^{3} \times 32$ lattice. In Fig. 2 we report the angular distribution of $C(\theta, r)$ for a distance $r=5.1 a$ from the static quark for the scalar diquark and the vector diquark with the $z$ component of the spin $J_{z}=1$. An enhancement of the distribution at $\cos \theta=0$ is evident for the scalar diquark,

\footnotetext{
${ }^{1}$ See http://qcd.nersc.gov.
} 


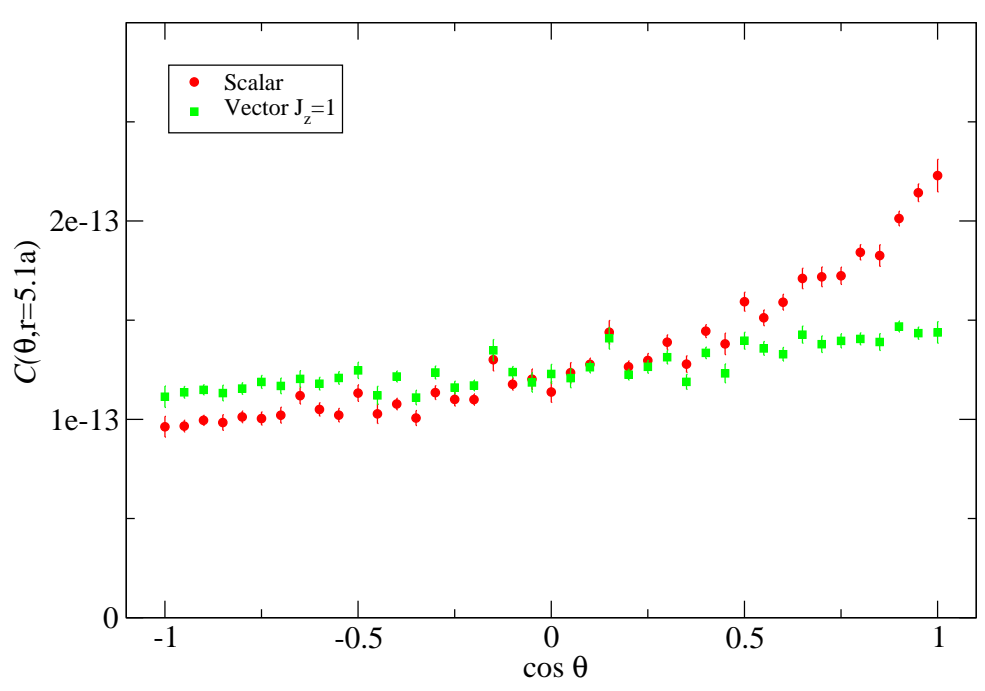

Figure 2: $C(\theta, r)$ versus $\cos \theta$ at distance $r=5.1 a$ from the static source.

while the distribution looks flatter for the vector diquark. A study of $C(r, \theta)$ at larger distances $r$ shows the same features. This confirms the expectation that the color field generated by the static source does not affect the physics of the diquark once $r$ is large enough, and shows that this asymptotic regime can be reached in our calculations. Hence the natural conclusion of this study is that a scalar diquark forms, while a vector diquark state, if it exists, is much broader. The densitydensity correlator technique allows also to give a definition of the size of the diquark. At fixed $r$ we can look at the correlator as a function of the $u-d$ separation $R_{u d}=2 r \sin (\theta / 2)$. An exponential behaviour $C\left(r, R_{u d}\right) \propto e^{-R_{u d} / R_{0}}$, as seen in ordinary hadrons [7], gives a gauge-invariant definition of the hadron size $R_{0}$. For the scalar diquark, our data are correctly described by an exponential ansatz over a wide range of $r$ (see Fig. 3 for an example). Results for $R_{0}$ as a function of $r$ are reported in Fig. 4. At small separations $r$ the value of $R_{0}$ depends on the radius $r$ of the shell. The extracted values of $R_{0}$ converge for $r \geq 4 a$. Once again, this confirms that the regime in which the physics of the diquark system is not disturbed by the field of the static source can be reached on our lattice. A safe estimate for the scalar diquark size is $R_{0}=0.92(9) \mathrm{fm}$. For the vector channel, the fitted size is noisier and seems to increase with $r$. The value at the maximal possible distance $r=8 a$ can be used to put a lower bound of 4.1(7) fm on the size of this diquark.

\section{Diquark masses}

From colour-spin effective hamiltonian arguments one can estimate the mass of the scalar diquark and the mass splitting $\Delta M=M_{\text {vector }}-M_{\text {scalar }}$ between the vector and the scalar diquark, obtaining respectively $M \simeq 320 \mathrm{MeV}$ and $\Delta M \simeq 200 \mathrm{MeV}$ [5]. Since we are using heavy quarks we expect these numbers to be different in our case. Again the colour-spin effective hamiltonian gives us a handle on what we should expect: if the mass of the degenerate constituent quarks increases, the mass $M$ of the scalar diquark increases and the mass splitting $\Delta M$ goes down as $1 / M^{2}$. Within our static-light baryon setup the mass of the diquark can not be accessed, since the mass of a 


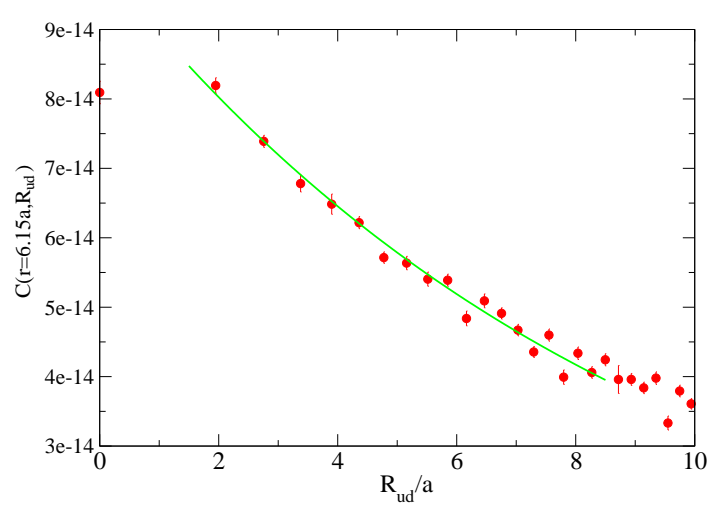

Figure 3: The exponential decay of $C(r=$ $\left.6.15 a, R_{u d}\right)$ at fixed $r$ for the scalar diquark (the fit is performed from $R_{u d}=2 a$ to $8 a$ ).

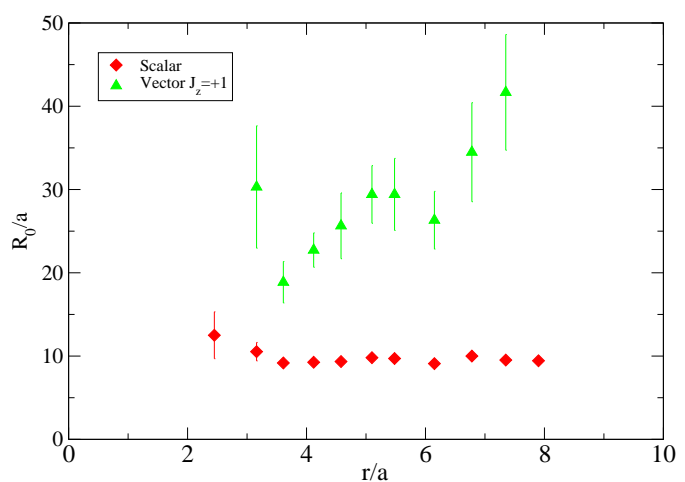

Figure 4: Fitted diquark size $R_{0}$ (in units of $a$ ) as a function of the distance $r / a$ of the diquark from the static quark.

hadron with a static quark is ultraviolet divergent. However, the mass difference can be extracted by looking at the temporal behaviour of the ratio of propagators of static-light baryons with the light quarks combined into the channels we are interested in.

\begin{tabular}{|c|c|c|c|}
\hline$\beta$ & $\kappa$ & $\Delta M(\mathrm{MeV})$ & $m_{\pi}(\mathrm{MeV})$ \\
\hline 6.0 & 0.1530 & 830 & $115(20)$ \\
5.8 & 0.1575 & 830 & $100(15)$ \\
5.8 & 0.1530 & 1000 & $67(7)$ \\
\hline
\end{tabular}

Table 2: Mass splittings for the scalar and the vector diquarks at the $\beta$ and $\kappa$ shown.

We study these ratios on a $16^{3} \times 32$ lattice at a given pion mass of about $830 \mathrm{MeV}$ at $\beta=6.0$ taking $\kappa=0.153$ and $\beta=5.8$ with $\kappa=0.1575$ to check scaling. At $\beta=5.8$ we also calculate $\Delta M$ at a larger pion mass of about $1 \mathrm{GeV}$ taking $\kappa=0.153$ to check the dependence of $\Delta M$ on the quark mass. We use 200 NERSC configurations for each value of $\beta$ and $\kappa$. To reduce the noise caused by the static source we used hypercubic smearing $[9,10]$. Our results are reported in Table 2 where the errors given are only statistical. Changing the fitting range we obtain values for $\Delta M$ that are consistent within these large statistical errors. The following features emerge: (a) at fixed $m_{\pi}$, the value of the mass splitting is independent of $\beta$, and this suggests that our calculation is reasonably free of lattice artifacts; (b) the mass splitting at $m_{\pi}=830 \mathrm{MeV}$ is smaller than the phenomenological estimate; (c) as $m_{\pi}$ is increased, the mass splitting decreases. (b) and (c) are compatible with qualitative expectations from colour-spin effective hamiltonian arguments above. Moreover, our results for the mass splittings are also compatible with a similar calculation reported at this conference [11], with estimates coming from an instanton liquid model [12] and with an earlier lattice calculation in Landau gauge [13] (see also [14]).

\section{Conclusions}

Density-density correlators in the background of a static source provide a gauge-invariant method for the study of diquark structure. Within this framework, we study the scalar and the vector diquark channels. Our results show that a diquark state forms in the scalar channel, with a size of $0.92(9) \mathrm{fm}$ (for a pion mass around $830 \mathrm{MeV}$ ). For the vector diquark we can provide a lower bound of 4.1(7) fm on its size. This is the first gauge invariant calculation from first principles that 
gives evidence for the existence of diquarks as colour antitriplet bound states of two quarks. The mass splitting between the scalar and vector diquarks is calculated; our results are compatible with extrapolations of phenomenological estimates towards large masses of the constituent quarks. Currently we are simulating at a third value of $\beta$ to check scaling and be able to study heavier quarks. At the same time we are increasing the statistics at $\beta=6.0$ and 5.8 by a factor of 10 . By using three values of quark masses we should be able to check the $1 / M^{2}$ scaling of the mass splitting between scalar and vector diquarks expected at heavy quark masses. Finally we are extending the study of the density-density correlation functions and of the mass splitting to the other diquark channels listed in Table 1.

\section{Acknowledgments}

We thank R. Jaffe and A. Polosa for useful discussions.

\section{References}

[1] M. Anselmino, E. Predazzi, S. Ekelin, S. Fredriksson, and D. B. Lichtenberg, Diquarks, Rev. Mod. Phys. 65 (1993) 1199-1234.

[2] M. G. Alford, K. Rajagopal, and F. Wilczek, Color-flavor locking and chiral symmetry breaking in high density QCD, Nucl. Phys. B537 (1999) 443-458, [hep-ph/ 9804403$].$

[3] R. L. Jaffe and F. Wilczek, Diquarks and exotic spectroscopy, Phys. Rev. Lett. 91 (2003) 232003, [hep-ph/0307341].

[4] L. Maiani, F. Piccinini, A. D. Polosa, and V. Riquer, A new look at scalar mesons, Phys. Rev. Lett. 93 (2004) 212002, [hep-ph/0 407017$].$

[5] R. L. Jaffe, Exotica, Phys. Rept. 409 (2005) 1-45, [hep-ph/ 0409065 ].

[6] J. W. Negele, Hadron structure in lattice qcd: Exploring the gluon wave functional, hep-lat/0007026.

[7] C. Alexandrou, P. de Forcrand, and A. Tsapalis, Probing hadron wave functions in lattice qcd, Phys. Rev. D66 (2002) 094503, [hep-lat/ 0206026 ].

[8] QCD-TARO Collaboration, S. Choe et al., Quenched charmonium spectrum, JHEP 08 (2003) 022, [hep-lat/0307004].

[9] A. Hasenfratz and F. Knechtli, Flavor symmetry and the static potential with hypercubic blocking, Phys. Rev. D64 (2001) 034504, [hep-lat/0103029].

[10] M. Della Morte, A. Shindler, and R. Sommer, On lattice actions for static quarks, JHEP 08 (2005) 051, [hep-lat/0506008].

[11] K. Orginos, Diquark properties from lattice qcd, PoS (LAT2005) (2005) 054.

[12] P. Faccioli, Studying diquark correlations in the nucleon by means of lattice qcd simulations, PoS (LAT2005) (2005) 052.

[13] M. Hess, F. Karsch, E. Laermann, and I. Wetzorke, Diquark masses from lattice qcd, Phys. Rev. D58 (1998) 111502, [hep-lat/9804023].

[14] R. Babich et al., Light hadron and diquark spectroscopy in quenched qcd with overlap quarks on a large lattice, hep-lat/0509027. 